\title{
Space environment during Solar Minimum of SC-23
}

\author{
Ahmed A. Hady \\ Astronomy and Meteorology Dept., Faculty of Sciences, Cairo University , \\ Giza, Egypt \\ email: aahady@cu.edu.eg
}

\begin{abstract}
Geomagnetic and solar storms and their occurrence rate with respect to the solar activity cycle is an important topic of space environment research. The minimum of solar activity during the solar cycle number 23 (SC-23) have detectable effects on the space environments, where the monthly mean of sunspot number and solar proton events effecting the space environment and produced many of Geophysical effects. A detailed study of the centers of activity produced the proton events are carried out. The electromagnetic emissions at the different bands during the impulsive phase of the flare are tabulated and discussed. The different Geophysical effects due to the events are studied. Conclusions about the proton solar events at minimum activity are presented.
\end{abstract}

Keywords. Deep solar minimum, Solar Activity, Climate change, global cooling.

The present paper examines the deep minimum of SC-23 and its potential impact on geomagnetic activity. In addition, a source region of the solar winds at solar activity minimum, especially in the solar cycle 23, the deepest during the last 100 years, has been studied. Is this episode comparable to the Maunder Minimum or is it like the Dalton Minimum?. Monthly and yearly means of sunspots during the SC-23 and its decline phase until December 2009 are given in Table (1). From Table (1) we note that the spotless days during years 2007. There were no sunspots observed over 147 days of the year's 365 days (41\%). During 2008, the spotless days were 266 of 366 days (73\% spotless days). During 2009 the spotless days were 260 of 365 days (71\% spotless days). The total spotless days during solar cycle 23 decline phase are 821 days, while the typical solar minimums were 486 days. Activity and timing of the current minimum, as well as the timing of the SC-24 maximum in 2013 compared with the start of the Dalton minimum de Toma et al. (2009).

Table (2), the daily Geomagnetic data of the Most eruptive days during the Peak period and decline period of Solar cycle 23. From this table, the geomagnetic data show detectable increases of recorded values during October - November 2003, compared with data during the period of SC-23. This increase has its peak during 29 November as in proton flounce case. This means that there are mutual effects of proton particle and the geomagnetic field (Pontieri et al. (2003)). There is a new deep minimum of SC-23 may extend through the next 30 year during the coming solar SC-23, 24, and 26, similar to what occurred during Dalton minimum era. There is notable increasing in the magnetic flux in the near space of the earth after high energetic solar activity during SC-23. 
Table 1 Monthly and yearly means of sunspot numbers of solar cycle 23, 24

Year 2001: Yearly Means: 110.58

$\begin{array}{lllllllllllll}\text { Monthly mean: } 95.6 & 80.6 & 113.5 & 10^{7} .7 & 96.6 & 134.0 & 81.8 & 106.4 & 150.7 & 125.5 & 106.5 & 132.2\end{array}$

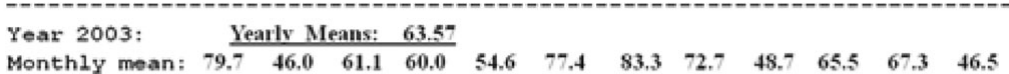

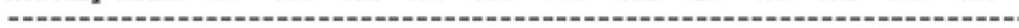

Year 2006: Yearly Means: 15.16

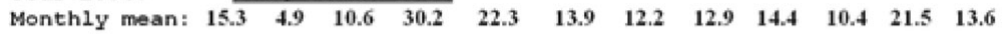

Year 2007: Yearly Means: 7.5

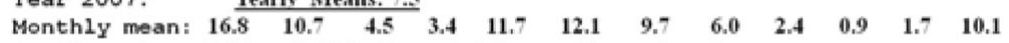

Spotless Days 149 of 365 days (41\% spotless days)

\begin{tabular}{llllllllllll} 
Year 2008: & \multicolumn{1}{l}{ Yearly Means: 2.85} \\
Monthly mean: 3.3 & 2.1 & 9.3 & 2.9 & 3.2 & 3.4 & 0.8 & 0.5 & 1.1 & 2.9 & 4.1 & 0.8
\end{tabular}

pot less Days 266 of 366 days ( 733 spot less days)

Year 2009: Yearly Means: 3.1, Start of Solar cycle 24, Jan. 2009)

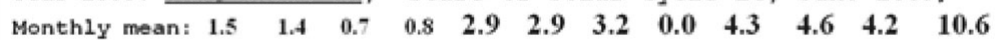

Spotless Days 260 of 365 days (71\% spotless days)

Year 2010: Yearly Means: 16.6

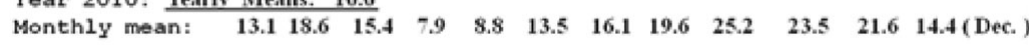

Spotless Days 51 of 365 days (14\% spotless davs)

Year 2011

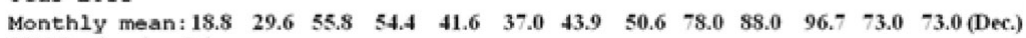
2011 Spotless days 2 days

Total spotless days Since 2004: 821 days (Typical Solar Min: 486 days)

Year 2012, monthly mean 58.3 (Jan) 33.1 (Feb) $64.2 \mathrm{Mar}) \quad 55.2(\mathrm{Apr}) \quad 55.2$ (May)

\begin{tabular}{|c|c|c|c|c|c|c|c|c|c|c|c|c|c|c|c|c|c|c|c|c|c|c|c|c|c|c|c|}
\hline \multirow{2}{*}{$\begin{array}{c}\text { Date } \\
20000714 \\
20000715\end{array}$} & \multicolumn{9}{|c|}{$\begin{array}{l}\text { Middle Latitude } \\
\text {-Frederickaburg- }\end{array}$} & \multicolumn{9}{|c|}{$\begin{array}{l}\text { High Latitude } \\
--- \text { College-- }\end{array}$} & \multicolumn{9}{|c|}{$\begin{array}{c}\text { Eatimated } \\
-- \text { Planetary-- }\end{array}$} \\
\hline & $\begin{array}{r}33 \\
148\end{array}$ & $\begin{array}{l}3 \\
3\end{array}$ & & $\frac{3}{3}$ & $\begin{array}{l}3 \\
3\end{array}$ & 6 & B & $\begin{array}{l}6 \\
9\end{array}$ & $\begin{array}{l}4 \\
9\end{array}$ & 49 & $\begin{array}{l}4 \\
3\end{array}$ & $\begin{array}{l}4 \\
4\end{array}$ & $\begin{array}{l}3 \\
5\end{array}$ & $\begin{array}{l}5 \\
6\end{array}$ & $\begin{array}{l}4 \\
7\end{array}$ & $\begin{array}{l}7 \\
-\end{array}$ & $\begin{array}{l}6 \\
-\end{array}$ & 4 & $\begin{array}{r}35 \\
152\end{array}$ & $\begin{array}{l}4 \\
4\end{array}$ & $\begin{array}{l}3 \\
4\end{array}$ & $\begin{array}{l}4 \\
5\end{array}$ & & $\begin{array}{l}4 \\
6\end{array}$ & $\begin{array}{l}6 \\
9\end{array}$ & & $\begin{array}{l}4 \\
5\end{array}$ \\
\hline 0001108 & 14 & 1 & 3 & 4 & 4 & 3 & 3 & 1 & 2 & - & - & - & - & - & - & - & - & - & 1.5 & 2 & 3 & 4 & & 3 & 3 & 1 & 3 \\
\hline 09 & 10 & 3 & 1 & 1 & 1 & 3 & $a$ & 2 & 3 & 21 & 2 & 2 & 1 & 3 & 5 & 5 & 4 & $a$ & 11 & $a$ & 1 & 1 & & 3 & $a$ & 3 & 3 \\
\hline $\begin{array}{l}24 \\
25\end{array}$ & $\begin{array}{r}5 \\
17\end{array}$ & $\begin{array}{l}3 \\
1\end{array}$ & $\frac{2}{2}$ & $\begin{array}{l}1 \\
2\end{array}$ & $\begin{array}{l}1 \\
0\end{array}$ & $\frac{1}{3}$ & $\frac{1}{0}$ & $\begin{array}{l}1 \\
4\end{array}$ & $\begin{array}{l}1 \\
6\end{array}$ & $\begin{array}{r}4 \\
23\end{array}$ & $\begin{array}{l}3 \\
1\end{array}$ & $\begin{array}{l}1 \\
2\end{array}$ & $\begin{array}{l}1 \\
2\end{array}$ & $\begin{array}{l}0 \\
2\end{array}$ & $\begin{array}{l}1 \\
0\end{array}$ & $\begin{array}{l}1 \\
0\end{array}$ & $\frac{1}{6}$ & 0 & $\begin{array}{r}6 \\
18\end{array}$ & $\begin{array}{l}3 \\
1\end{array}$ & $\begin{array}{l}1 \\
2\end{array}$ & $\frac{1}{2}$ & & $\begin{array}{l}2 \\
1\end{array}$ & $\begin{array}{l}2 \\
2\end{array}$ & 2 & 1 \\
\hline 04 & 4 & 0 & 0 & 2 & 3 & 1 & 2 & 1 & 0 & 17 & 0 & 0 & 3 & 5 & 5 & 3 & 2 & & 7 & 0 & 0 & 2 & & 3 & 3 & 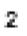 & 1 \\
\hline 20011105 & 12 & 0 & 0 & 1 & 2 & 4 & 3 & 4 & 3 & 11 & 0 & 0 & 0 & 2 & 3 & 4 & 4 & & 13 & 0 & 0 & 1 & 4 & 3 & 4 & 5 & 4 \\
\hline 122 & $B$ & 1 & 2 & 2 & 2 & 2 & 2 & 3 & 2 & - & 0 & 2 & 2 & - & 2 & 2 & 2 & 1 & 8 & 1 & 2 & 2 & & 2 & 2 & $d$ & 2 \\
\hline 23 & 11 & 2 & 1 & 2 & 2 & 3 & 4 & 1 & 3 & 23 & 3 & 2 & 3 & 4 & 5 & 5 & 2 & & 12 & 3 & 2 & 2 & & 3 & 3 & $d$ & $d$ \\
\hline 20020421 & 4 & 1 & 1 & 1 & 1 & 1 & 1 & 2 & 2 & 7 & 3 & 2 & 2 & 0 & 1 & 2 & 2 & 2 & 7 & 3 & 2 & 2 & 1 & 2 & 2 & 2 & 3 \\
\hline 28 & 15 & 2 & 4 & 3 & 3 & 2 & 3 & 3 & 3 & 28 & 2 & 3 & 5 & 5 & 3 & 5 & 4 & 3 & 20 & 3 & 4 & 4 & & 3 & 4 & 3 & 4 \\
\hline 029 & 199 & 3 & 3 & 9 & 6 & 7 & 7 & 9 & 9 & 97 & 5 & 4 & 9 & 8 & 7 & 8 & 9 & & 189 & 4 & 3 & 9 & & 7 & 7 & 9 & 8 \\
\hline 20031030 & 144 & 7 & 5 & 4 & 4 & 4 & 6 & 9 & 9 & 124 & 6 & 5 & 4 & 6 & 7 & 8 & 7 & 8 & 162 & 8 & 7 & 6 & 5 & 5 & 8 & 9 & 9 \\
\hline 20031031 & 73 & 8 & 7 & 6 & 4 & 5 & 3 & 3 & 3 & 69 & 6 & 6 & 6 & 6 & 6 & 5 & 6 & & 93 & 8 & 7 & 7 & 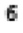 & 6 & 5 & 4 & 4 \\
\hline 20031101 & 16 & 5 & 4 & 3 & 1 & 2 & 2 & 1 & 3 & 32 & 4 & 4 & 5 & 5 & 5 & 4 & 3 & & 21 & 4 & 5 & 4 & 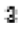 & 3 & 3 & 3 & 3 \\
\hline 20031102 & 11 & 3 & 3 & 2 & 2 & 2 & 3 & 3 & 2 & 27 & 4 & 3 & 3 & 4 & 5 & 5 & 4 & & 18 & 3 & 4 & 3 & 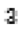 & 3 & 4 & 4 & 3 \\
\hline 200 & 15 & 4 & 2 & 1 & 1 & 5 & 2 & 2 & 3 & 12 & 3 & 3 & 1 & 3 & 2 & 3 & 2 & & 10 & 3 & 3 & 2 & a & 2 & 3 & 2 & 3 \\
\hline 20031104 & 20 & 2 & 2 & 5 & 5 & 2 & 2 & 4 & 2 & 34 & 3 & 3 & 5 & 7 & 3 & 2 & 4 & & 31 & 3 & 2 & 5 & $\gamma$ & $a$ & 3 & 4 & 3 \\
\hline
\end{tabular}

\section{References}

de Toma, G., Gibson, S., Emery, B., \& Kozyra, J. 2009, AIP Conf. Proc. 2009; 1216: 667-670 Pontieri A., F. Lepreti, L. sorriso-Volvo, A., \& Vecchio, V. Carbone, 2003, Solar physics 213:195201 